\title{
Effect of Lateral Ankle Ligament Anesthesia on Single-Leg Stance Stability
}

\author{
BRYAN L. RIEMANN ${ }^{1,2}$, JOSEPH B. MYERS ${ }^{1}$, DAVID A. STONE ${ }^{1}$, and SCOTT M. LEPHART ${ }^{1}$ \\ ${ }^{I}$ Neuromuscular Research Laboratory, Department of Orthopaedic Surgery, University of Pittsburgh, Pittsburgh, PA; and \\ ${ }^{2}$ Jiann-Ping Hsu School of Public Health, Georgia Southern University, Statesboro, GA
}

\begin{abstract}
RIEMANN, B. L., J. B. MYERS, D. A. STONE, and S. M. LEPHART. Effect of Lateral Ankle Ligament Anesthesia on Single-Leg Stance Stability. Med. Sci. Sports Exerc., Vol. 36, No. 3, pp. 388-396, 2004. Purpose: This study was designed to determine, in isolation, the contribution of lateral ankle ligament mechanoreceptors to postural stability during single leg static (eyes open, eyes closed) and landing tasks. Methods: Fourteen healthy subjects (nine males, five females) underwent two different treatment conditions (control, anesthesia) in a counterbalanced order (48-h interval). During the anesthetic treatment, lidocaine was injected into the anterior talofibular and calcaneofibular ligament $(1.5 \mathrm{cc}$ each) regions. Postural stability was measured using forceplate and kinematic variables. The average of each variable across multiple trials under each treatment for the three tasks was analyzed statistically. Results: Results of all statistical analyses failed to demonstrate significant alterations $(P>0.05)$ in postural control attributable to the treatment condition. Conclusion: These results suggest that lateral ankle ligament mechanoreceptors either do not make a significant contribution to single leg stance stability, do not have a unique, irreplaceable role, or have a role that is too subtle to be detected given the measurement techniques used. Thus, the idea that single leg stability becomes altered after ankle joint injury because of proprioceptive disruption was not supported. This would suggest that reported postural control alterations in persons after repetitive ankle injury more likely occur as a result of alterations in mechanical stability, motor components, and/or central motor programming. Key Words: PROPRIOCEPTION, ANKLE INSTABILITY, BALANCE, EQUILIBRIUM
\end{abstract}

$\mathrm{D}$ espite the lack of understanding concerning the significance of articular mechanoreceptor information to postural control, as well as the numerous reports of orthopedic injury adversely affecting both sensory and motor components of the body $(16,17,30)$, the orthopedic community has largely considered postural control from only an afferent perspective $(9,11,14,28)$. Interest began with Freeman (9), who took the principles of Rhomberg and directly applied them to an orthopedic pathology, functional ankle instability. Specifically, these investigators made bilateral comparisons of single leg postural stability (eyes closed) in patients with unilateral pathology and attributed the decreases in postural stability while standing on the involved limb as being indicative of proprioceptive disturbances. Tropp et al. (28), about 20 yr later, combined the ideas of Rhomberg and Freeman et al. with stabilimetry, the technique of using force platforms to objectively measure the excursion of the center of pressure during an assessment. Measuring postural stability prospectively before a soccer season, they were able to demonstrate that players who had

\footnotetext{
Address for correspondence: Bryan Riemann, Ph.D., ATC, Graduate Athletic Training Program, Georgia Southern University, P.O. Box 8076, Statesboro, GA 30460; E-mail: briemann@georgiasouthern.edu. Submitted for publication April 2003.

Accepted for publication October 2003.
}

0195-9131/04/3603-0388

MEDICINE \& SCIENCE IN SPORTS \& EXERCISE E

Copyright (C) 2004 by the American College of Sports Medicine

DOI: 10.1249/01.MSS.0000117131.93989.9B poor postural stability ran a higher risk than normals for sustaining an ankle injury during the season. Since their report, many other investigators have used similar techniques. Although some of these studies have found alterations $(11,29)$, others have not $(1,14)$. Understanding the role of articular receptors in postural control may help to explain some of these controversial results, as well as provide a more objective basis to many commonly used clinical rehabilitation procedures (23).

Only few investigations have focused solely on the function of ankle articular receptors in postural control $(3,12)$. Hertel et al. (12) compared the excursion and mean location of the center of balance (pressure) during single leg stance (eyes closed) under normal conditions and after lateral ankle anesthesia. The results of the study demonstrated that after anesthesia subjects altered their mean center of balance laterally during static conditions and medially during dynamic (slow platform tilt) conditions. Limitations in the forceplate apparatus prevented these investigators from considering corrective actions against the support surface (shear forces), as well as occurrence of proximal joint (knee, hip, trunk) movement patterns. DeCarlo and Talbot (3) considered the contribution of the lateral ankle mechanoreceptors to multiaxial platform stability. The results of their investigation revealed increased stability after anesthesia, most likely arising as a result of learning effects associated with repeated exposures to the test.

Thus, the question concerning the importance and role of proprioception from articular mechanoreceptors still remains unanswered. The current investigation was designed with the intent of determining, in isolation, the contribution 
of lateral ankle ligament mechanoreceptors to single stance stability during both static and dynamic tasks.

\section{METHODS}

Subjects. Fourteen recreationally active individuals (nine males, five females, height $=175.4 \pm 8.9 \mathrm{~cm}$, mass $=72.6 \pm 11.7 \mathrm{~kg}$, age $=24.2 \pm 3.3 \mathrm{yr}$ ) were used in this investigation based on an a priori power analysis of the treatment effects reported by Hertel et al. (12). Recreationally active was operationally defined as participation in some form of physical activity $20 \mathrm{~min}$ in duration at least $3 \times \mathrm{wk}^{-1}$. All participants had no history of previous ankle, knee, or hip musculoskeletal pathology that could affect their ability to perform single leg postural control tests. Additionally, subjects were screened for recent head injuries and any preexisting visual, vestibular, and/or balance disorders by asking about any previously diagnosed conditions.

Procedures. A true experimental crossover design was used, whereby each participant underwent two treatment conditions (normal, lateral ankle anesthesia) with a minimum intertest interval of $48 \mathrm{~h}$. After each treatment, participants underwent two static postural stability tasks, eyes open (SLEO) and eyes closed (SLEC), and one dynamic single leg landing task (SLLand). To assist in controlling for learning effects, subjects were randomly assigned to a counterbalanced schedule of treatment and task testing order. Before a subject's first testing session, they were required to attend a 20-min introductory session to become familiar with the testing tasks. Additionally, subjects were asked to read and sign an Institutional Review Board approved consent form at the introductory session. All treatments and testing were conducted with the subjects barefoot using the dominant limb, defined as the preferred leg to kick a ball.

Lateral ankle joint anesthesia was attained using similar methods as previously reported (18). Anesthetic 1\% lidocaine (preservative and epinephrine free) (Elkins-Sinn, Inc., Cherry Hill, NJ) was injected by a board certified physician into the anterior talofibular and calcaneofibular ligament regions (1.5 cc each site). Induction time for anesthetic effects was estimated to fully occur within $20 \mathrm{~min}$ after injection and lasted approximately $3-6 \mathrm{~h}$. During the control treatments, subjects were asked to remain seated for $20 \mathrm{~min}$ to control for the potential confounding effect of time associated with administration of lateral ankle ligament anesthesia.

Static postural stability testing, SLEO and SLEC, was conducted with the participant standing on a force platform. Each subject performed three trials of each task (SLEO, SLEC), with one practice attempt of both tasks before the scored trials. Each trial lasted $12 \mathrm{~s}$ in duration with the stance foot placed such that the long axis of the foot was aligned with the short axis of the forceplate. Participants were instructed to maintain their hands on the iliac crests and the contralateral limb (nonstance limb) in $30^{\circ}$ of knee and hip flexion (Fig. 1). Progressive directions were given to the participants to first take the required stance position, and then place their hands on the iliac crest (followed by eye

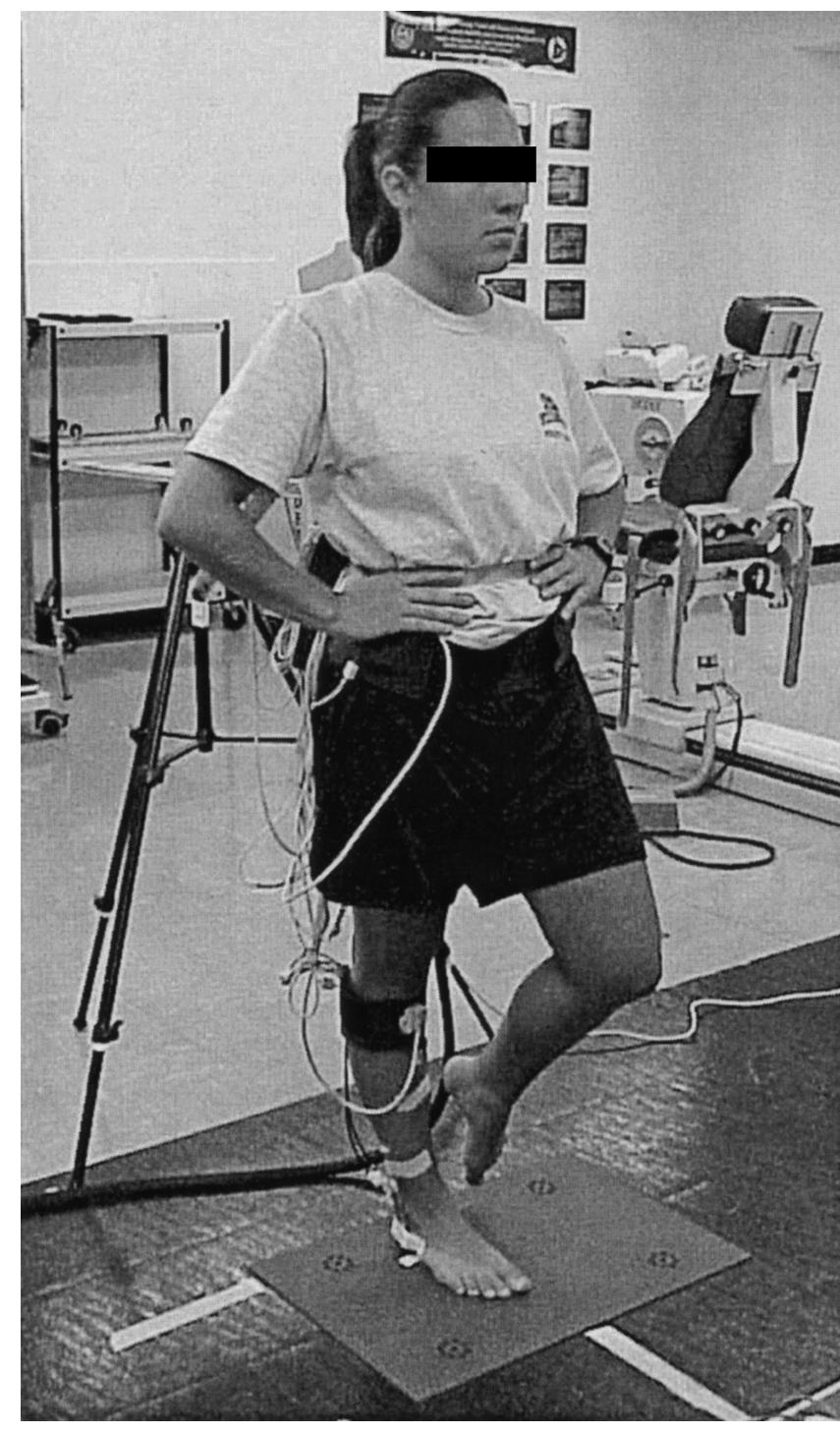

FIGURE 1-Standardized testing position for the SLEO and SLEC tasks. Illustrated is the SLEO task.

closure during eyes closed trials). Data collection for the $12 \mathrm{~s}$ began upon a preset signal given by the participant. Participants were instructed to attempt to remain as motionless as possible and that upon losing their balance they were to make the necessary adjustments (i.e., hands off iliac crests, touch down) with the goal of returning to the standardized testing position as quickly as possible. In the event that a compensatory event occurred, defined according to the Balance Error Scoring System (24), the principal investigator activated an analog switch synchronized with the kinematic data for the duration of time the participant remains out of the standardized testing position. For each incomplete trial, defined as those in which more than three compensatory events occurred, participants were given one retest trial. Thus, the maximum number of trials a subject could attempt at a given condition was six (26).

The SLLand was conducted from a step height of $15 \mathrm{~cm}$. Subjects were instructed to begin with a single leg stance on the dominant limb (test limb), first toe at the edge of the 
step, and with their hands on the iliac crests (Fig. 2). The subject was asked to hop off the step and land onto the forceplate using only the test limb. A target (X) was placed $30 \mathrm{~cm}$ horizontally from the base of the step. On landing, participants were asked to control their balance to remain in a single leg stance position for $3 \mathrm{~s}$ with the hands remaining on the iliac crests and the head level and facing forward. Participants were given one practice attempt before completing three test trials. Successful trials were defined as maintaining the hands on the iliac crests and not touching down with the contralateral limb during all parts of the test and successfully covering the target. For each incomplete trial, participants were given one retest trial.

Kinematic data collection. Similar to another report published by our laboratory (26), kinematic measurements of ankle, knee, hip, and trunk were made with an electromagnetic kinematic tracking system (Motion Monitor, Innovative Sports Training, Inc., Chicago, IL). The system consists of a standard range transmitter (Motion Star, Ascension Technology Corp., Burlington, VT) with four tethered receivers (sensors) that convey the signals to a base computer. Sensor data were collected at $100 \mathrm{~Hz}$ with all hardware settings in the default mode. The Motion Monitor software was used to calculate sensor position and orientation from the data conveyed by the sensors. During subject setup, sensors were firmly secured to the lower leg $(35 \mathrm{~cm}$ above the inferior tip of the lateral malleolus), anterior upper leg (10 $\mathrm{cm}$ above knee joint line), base of the sacrum, and upper trunk (C7-T1 junction). The ankle, knee, and hip joint centers with respect to fixed sensors were calculated by taking the midpoint between two points digitized by an additional movable sensor on contralateral aspects of the joint. Participant height and weight were used for the appropriate anthropometric calculation required for locating each segment's center of mass using the Dempster codes as
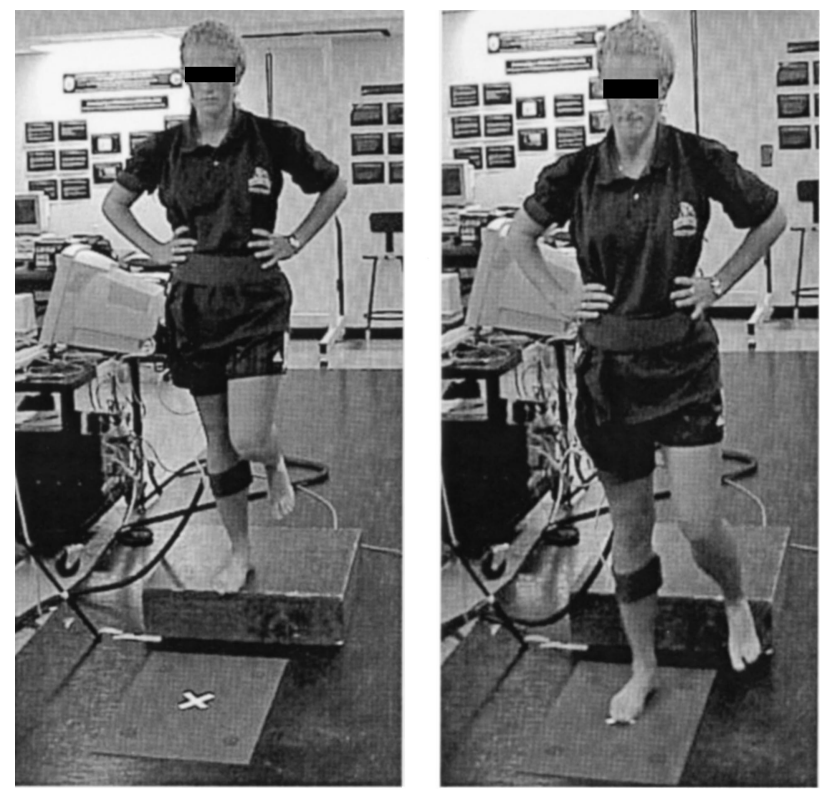

a.

b.

FIGURE 2-Starting (a) and ending (b) positions for the SLL and task. reported by Winter (31). Additionally, $5 \mathrm{~s}$ of calibration data were collected with the subject standing in anatomical position before any treatment or testing procedures.

During each task, the participants were aligned with a global axes system established with respect to the transmitter. Local coordinate systems for the shank, thigh, pelvis, and trunk were established according to the International Society of Biomechanics standardization recommendation. Euler angles (Zy' $\left.\mathrm{x}^{\prime \prime}\right)$ were calculated between the shank and thigh segments and the thigh and trunk segments to determine the relative knee and hip-trunk joint angles, respectively. The specific joint movements considered included knee flexion, abduction and rotation as well as hip-trunk flexion, abduction (i.e., lateral flexion), and rotation.

Because of the metallic forceplate used in the investigation, we could not capture ankle kinematics directly. Rather, ankle motion was indirectly determined based on shank motion, during separate subsequent analyses. The segment axes of the shank were aligned according to the global axes for the respective task. By using these axes alignments, shank orientation in the plane created by the anterior and superior global axes represented ankle dorsiflexion-plantar flexion, shank orientation in the plane created by the right and superior global axes represented inversion-eversion, and shank orientation in the plane created by the anterior and right global axes represented foot abduction-adduction. The calculations of the shank orientation about the three axes were determined using Euler's angles, using a flexion-extension, abduction-adduction, and inversion-eversion sequence. The comparison of this method in determining ankle kinematics to direct measurements was recently made (27) and discussed in the context of a similar investigation (26). Specifically, strong cross-correlations between respective shank orientation and direct inversion/eversion (SLEO $=-0.91$, SLEC $=-0.97)$ and plantar flexion/dorsiflexion $($ SLEO $=-0.75$, SLEC $=-0.76)$ measurements were demonstrated (27).

Forceplate data collection. A forceplate (Model K80801, Type 4060, Bertec Corporation, Columbus, OH) was used to collection ground reaction force data during all tasks. Forceplate signals were amplified (AM-7600 amplifier, Bertec Corporation, Columbus, $\mathrm{OH}$ ), filtered (four pole filter, $500-\mathrm{Hz}$ cutoff) and digitized using an analog to digital card (ComputerBoard DAS 1602-12, ComputerBoards, Inc., Middleboro, MA). During the SLEO and SLEC tasks, the forceplate data were acquired with a sampling frequency of $100 \mathrm{~Hz}$, whereas during the SLLand the sampling rate was increased to $1000 \mathrm{~Hz}$. Signals from the forceplate were converted to force and moment components, and for the SLEO and SLEC trials the center of pressure was calculated.

Data reduction. The angular kinematic data for all tasks and the forceplate data for the SLEO/SLEC tasks were smoothed (fourth-order zero phase lag Butterworth) with a $10-\mathrm{Hz}$ cutoff, whereas the forceplate data from the SLLand was smoothed (fourth-order zero phase lag Butterworth) with a $60-\mathrm{Hz}$ cutoff.

For the SLEO/SLEC tasks, although $12 \mathrm{~s}$ of kinematic and forceplate data were recorded, only the middle $10 \mathrm{~s}$ 
were used to calculate the variables of interest. First, intervals of time in which a compensatory event occurred, as indicated by the electronic switch signal were truncated. For each of the joints, ankle, knee, and hip-trunk, the vector sum of the three separate angular position vectors (flexion-extension, abduction-adduction, and rotation) were calculated at each sampling instance. Next, the difference between two successive vectors sums was calculated and averaged across the entire trial. The average difference at a particular joint across a trial represented the magnitude of the average angular distance (i.e., corrective action) between sampling instances independent of mean joint position (26). The three average angular distances from the ankle, knee, and hiptrunk were used as dependent variables entered into the statistical analyses. The absolute and relative reliability of these methods have been previously reported by our laboratory (26). From the center of pressure data during SLEO and SLEC trials, the average distance from mean center of pressure location $\left(\mathrm{COP}_{\text {Dist }}\right)$ was calculated. This variable indicates on average how far the center of pressure deviates from a mean position independent of direction and was recently reported to correlate highly with a respective center of mass variable during single leg stance (25). Lastly, the average vertical distance of the body's center of mass with respect to the center of the ankle joint during each trial $\left(\mathrm{COM}_{\text {height }}\right)$, normalized to the anatomical stance (calibration) position, was calculated. The kinematic and anthropometric data from the four body segments considered (shank, thigh, pelvis, and trunk) were used to calculate the center of mass location.

After ground contact during the SLLand task, the time to attain stabilization $\left(\mathrm{STAB}_{\text {Time }}\right)$ was calculated. This variable was determined by vector summing the three ground reaction forces recorded by the forceplate into a single composite vector. Time to attain stabilization was operationally defined by using a sequential estimation algorithm to determine the time when the sequential average of the composite vector remained within one-quarter standard deviation of the overall series mean for the $3 \mathrm{~s}$ after ground contact. This algorithm has been used in a similar task application by Colby et al. (2). Within $\mathrm{STAB}_{\text {Time }}$, the average angular displacements of the ankle, knee, and hiptrunk joints and $\mathrm{COM}_{\text {height }}$ were calculated exactly as described for the SLEO and SLEC tasks. Lastly, to determine whether the participants differed in jump and landing technique, the peak vertical ground reaction force $\left(\mathrm{vGRF}_{\text {Peak }}\right)$ normalized to body weight, time to $\mathrm{vGRF}_{\text {Peak }}\left(\mathrm{vGRF}_{\text {Time }}\right.$ Peak) and knee and hip-trunk joint angles (flexion and abduction) at ground contact were determined.

Statistical analyses. The average of each dependent variable across the multiple trials of each task (SLEO, SLEC, and SLLand) were calculated and separately entered into the statistical analyses. Repeated measures analyses of variance (RMANOVA) were conducted on all variables using the Statistical Package for Social Sciences Version 11.5 (SPSS, Inc., Chicago, IL).

The dependent variables of $\mathrm{COP}_{\text {Dist }}, \mathrm{COM}_{\text {height }}$, time of first compensatory event, number of compensatory events, and total time of compensatory events for the SLEO and SLEC tasks were analyzed similarly using treatment and task as within-subject factors. Joint, with three levels (ankle, knee, and hip-trunk), was added as a third factor during the statistical analysis of the angular displacement dependent variables.

A single within-subject factor, treatment, was used for statistical analysis of $\mathrm{STAB}_{\text {Time }}, \mathrm{COM}_{\text {height }}, \mathrm{vGRF}_{\text {Peak }}$, and $v G_{\text {Time Peak }}$ from the SLLand. Similar to the above tasks, joint was added as an additional factor during the analyses involving the kinematic variables. For angle at ground contact, there were two levels of joint (knee and hip-trunk), whereas for the angular displacement variables there were three levels of joint (ankle, knee, and hip-trunk).

\section{RESULTS}

SLEO and SLEC. Descriptive statistics for the dependent variables of the SLEO and SLEC tasks are given in Tables $1-4$. There were no significant differences $(P>$ 0.05 ) with respect to the number of compensatory events, time to the first compensatory event, and total time of compensatory events during the lateral ankle anesthesia condition in comparison with the control condition as evidenced by the condition by vision interaction and main effect for condition statistical tests. Significant main effects for vision (SLEO > SLEC) were revealed for the number of compensatory events $\left(F_{(1,13)}=9.84, P=0.008\right)$ and time to first compensatory event $\left(F_{(1,13)}=16.134, P=0.001\right)$. The difference in the total time of compensatory events between SLEO and SLEC approached significance $\left(F_{(1,13)}=3.71, P\right.$ $=0.076$ ).

The participants did not alter their $\mathrm{COM}_{\text {height }}$ with respect to ankle joint between the two treatment conditions $\left(F_{(1,12)}\right.$ $=0.202, P=0.661)$ nor between SLEO and $\operatorname{SLEC}\left(F_{(1,12)}\right.$ $=0.787, P=0.392)$. The condition by vision interaction for $\mathrm{COM}_{\text {height }}$ was also not significant $\left(F_{(1,12)}=0.835, P=\right.$ 0.379).

Compared with the control condition, lateral ankle anesthesia had no effect on the average angular displacement magnitudes as evidenced by all interactions involving condition (condition $\times$ vision $\times$ joint: $F_{(2,26)}=0.269, P=$ 0.766 ; condition $\times$ joint: $F_{(2,26)}=0.453, P=0.641$; condition $\times$ vision: $\left.F_{(1,13)}=0.005, P=0.944\right)$, as well as the main effect for condition $\left(F_{(1,13)}=0.313, P=0.585\right)$. The magnitude of the average angular displacement was significantly different between several of the vision-joint combinations $\left(F_{(2,26)}=41.2, P<0.001\right)$ (Fig. 3). Post hoc comparisons within both visual conditions yielded the quan-

TABLE 1. Means $( \pm S D)$ for the compensatory event variables during SLEO and SLEC.

\begin{tabular}{llllll}
\hline & \multicolumn{2}{c}{ Eyes Open } & & \multicolumn{2}{c}{ Eyes Closed } \\
\cline { 2 - 3 } \cline { 5 - 6 } \cline { 5 - 6 } & Control & Anesthesia & & Control & Anesthesia \\
\hline Time to first $(\mathrm{s})^{*}$ & 0.000 & 0.000 & & $1.65 \pm 1.90$ & $1.04 \pm 2.02$ \\
$N^{*}$ & 0 & 0 & & $0.43 \pm 0.44$ & $0.36 \pm 0.53$ \\
Length (s) & 0 & 0 & & $0.19 \pm 0.20$ & $0.14 \pm 0.54$ \\
\hline
\end{tabular}

* Eyes open significantly less than eyes closed ( $P=0.001$; main effect for vision). 
TABLE 2. Means $( \pm S D)$ for the normalized vertical distance relative to the ankle joint during SLEO and SLEC.

\begin{tabular}{lcclcc}
\hline & \multicolumn{2}{c}{ Eyes Open } & & \multicolumn{2}{c}{ Eyes Closed } \\
\cline { 2 - 3 } \cline { 5 - 6 } & Control & Anesthesia & & Control & Anesthesia \\
\hline $\mathrm{COM}_{\text {Height }}(\%)$ & $99.6 \pm 1.6$ & $101 \pm 3.7$ & & $99.6 \pm 1.8$ & $99.2 \pm 6.2$ \\
\hline $\mathrm{COM}_{\text {Height }}$, normalized vertical center of mass distance from ankle joint.
\end{tabular}

tity of corrective action at the knee during SLEC to be significantly less than that observed at the ankle and hiptrunk. Post hoc comparisons within each joint between the visual conditions revealed the significantly greater corrective action occurring during SLEO than SLEC for all three joints. Lastly, the main effect for joint $\left(F_{(2,26)}=27.9, P<\right.$ $0.001)$ revealed significantly greater corrective action occurred at the ankle and hip-trunk compared with the knee, whereas the main effect for vision revealed that significantly greater average angular displacement occurred during SLEC compared with $\operatorname{SLEO}\left(F_{(1,13)}=93.0, P<0.001\right)$.

Similar to the average angular displacement, the lateral ankle anesthesia had no significant effect on $\mathrm{COP}_{\text {Dist }}$ as evidenced by the condition by vision interaction $\left(F_{(1,13)}=\right.$ $0.728, P=0.409)$ and main effect for condition $\left(F_{(1,13)}=1.51\right.$, $P=0.241) . \mathrm{COP}_{\text {Dist }}$ was significantly greater during SLEC than $\operatorname{SLEO}\left(F_{(1,13)}=146.17, P<0.001\right)$.

SLLand. Descriptive statistics for the dependent variables for the SLLand task are given in Tables 5 and 6. The landing techniques used by the participants were similar between the lateral ankle anesthesia and control conditions. The knee and hip-trunk flexion angles at ground contact were not significantly different between the conditions (interaction: $F_{(1,13)}=0.805, P=0.386$; main effect: $F_{(1,13)}=$ 2.62, $P=0.130)$. Comparison between the knee and hiptrunk joint $\left(F_{(1,13)}=505.11, P<0.000\right)$ revealed a greater flexion angle in the latter compared with the former joint. The $\operatorname{vGRF}_{\text {Peak }}\left(F_{(1,13)}=0.451, P=0.513\right)$ and $\operatorname{vGRF}_{\text {Time }}$ Peak $\left(F_{(1,13)}=1.98, P=0.183\right)$ were not significantly different between the treatment conditions.

$\mathrm{STAB}_{\text {Time }}$ was $1.115 \pm 0.080 \mathrm{~s}$ and $1.143 \pm 0.082 \mathrm{~s}$ for the control and lateral ankle anesthesia conditions, respectively; however, the difference was not statistically significant $\left(F_{(1,13)}=2.26, P=0.156\right)$. Lateral ankle anesthesia had no effect on the quantity of joint corrective action occurring during $\mathrm{STAB}_{\text {Time }}$ as evidenced by a nonsignificant condition by joint interaction $\left(F_{(2,26)}=0.417, P=\right.$ $0.663)$ and main effect for condition $\left(F_{(1,13)}=0.020\right.$, $P=0.889)$. The main effect for joint was significant $\left(F_{(2,26)}\right.$ $=148.85, P<0.001)$, with post hoc comparisons revealing the quantity knee corrective action to be greater than ankle, which in turn was significantly greater than the quantity

TABLE 3. Means ( \pm SD) for joint corrective action (deg) during SLEO and SLEC.

\begin{tabular}{lccccc}
\hline & \multicolumn{2}{c}{ Eyes Open* } & & \multicolumn{2}{c}{ Eyes Closed } \\
\cline { 2 - 3 } \cline { 5 - 6 } & Control & Anesthesia & & Control & Anesthesia \\
\hline Ankle & $0.0144 \pm 0.0071$ & $0.0160 \pm 0.0102$ & & $0.0722 \pm 0.0308$ & $0.0718 \pm 0.0407$ \\
Knee† & $0.0096 \pm 0.0030$ & $0.0111 \pm 0.0048$ & & $0.0346 \pm 0.0142$ & $0.0374 \pm 0.0154$ \\
Hip & $0.0148 \pm 0.0094$ & $0.0727 \pm 0.0279$ & & $0.0181 \pm 0.0124$ & $0.0780 \pm 0.0378$ \\
\hline * Eyes open significantly less than eyes closed $(P<0.001 ;$ main effect for vision). \\
† Knee significantly less than ankle and hip $(P<0.001$; main effect for joint).
\end{tabular}

TABLE 4. Means $( \pm S D)$ for the average distance from mean center of pressure location during SLEO and SLEC.

\begin{tabular}{lcccc}
\hline & \multicolumn{2}{c}{ Eyes Open* } & \multicolumn{2}{c}{ Eyes Closed } \\
\cline { 2 - 4 } & Control & Anesthesia & Control & Anesthesia \\
\hline $\mathrm{COP}_{\text {Dist }}(\mathrm{m})$ & $0.0054 \pm 0.0010$ & $0.0056 \pm 0.0012$ & $0.0126 \pm 0.0025$ & $0.0135 \pm 0.0033$ \\
\hline COP $_{\text {Dist, }}$ average distance from mean center of pressure location. \\
* Eyes open significantly less than eyes closed $(P<0.001$; main effect for vision).
\end{tabular}

occurring at the hip-trunk joint. Finally, similar to SLEO and SLEC, the participants did not alter their $\mathrm{COM}_{\text {height }}$ under the lateral ankle anesthesia in comparison to the control condition $\left(F_{(1,12)}=1.16, P=0.301\right)$.

\section{DISCUSSION}

The most remarkable discovery in this investigation was the failure of the lateral ankle ligament anesthesia to induce significant changes in postural control across all dependent variables in comparison with the control condition. The only significant results revealed in this investigation were related to vision during the static tasks and joint during all three tasks. The lack of significant alterations after the anesthetic treatment challenges the traditional orthopedic perspective that postural control becomes altered after injury solely because of articular mechanoreceptor losses. As stated earlier, although several studies have reported disrupted postural control after ligamentous injury, an equal number of studies have failed to demonstrate differences (23). The nonsignificant outcomes of all dependent variables attributable to the treatment condition in the current investigation suggest that the altered postural control after orthopedic injury reported previously are more likely a result of mechanical stability losses, central nervous system modifications, and/or motor systems deficits. In other words, based on the results of the present study, it is highly unlikely that losses of mechanoreceptor inputs alone could explain the alterations reported in the previous literature. Similarly, based on the results of the present investigation, it is speculated that the proximal joint compensations reported previously $(10,21,29)$ in patients with ankle instability were

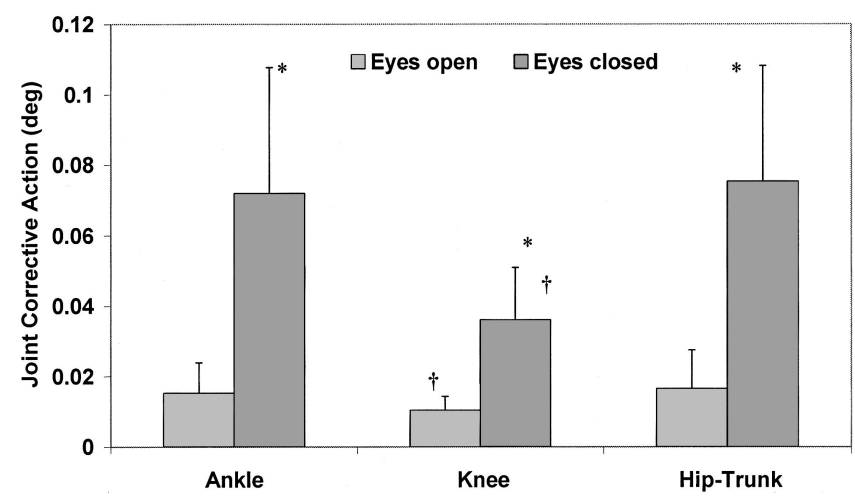

FIGURE 3-Comparison of joint corrective action between joints and visual conditions. Data are means \pm SD. ${ }^{*}$ Eyes open significantly less than eyes closed within each joint $(P<0.05)$; $†$ knee corrective action significantly less than ankle and hip-trunk within each visual condition $(P<0.05)$. 
TABLE 5. Means $( \pm S D)$ for dependent variables concerning joint angles at ground contact and vertical ground reaction force characteristics during SLLand.

\begin{tabular}{lccccc}
\hline & \multicolumn{2}{c}{ Joint Angle at GC $\left(^{\circ}\right)$} & & \multicolumn{2}{c}{ vGRF } \\
\cline { 2 - 3 } \cline { 5 - 6 } & Knee & Hip-Trunk $^{*}$ & & Peak (BW) & $\begin{array}{c}\text { Time to Peak } \\
\text { (s) }\end{array}$ \\
\hline Control & $-22.54 \pm 6.69$ & $33.29 \pm 6.52$ & & $2.52 \pm 0.22$ & $0.105 \pm 0.009$ \\
Anesthesia & $-23.24 \pm 5.43$ & $30.68 \pm 6.76$ & & $2.50 \pm 0.22$ & $0.102 \pm 0.011$ \\
\hline
\end{tabular}

GC, ground contact; vGRF, vertical ground reaction force; BW, body weight units.

* Hip-trunk flexion greater than knee ( $P<0.001$; main effect for joint).

likely attributable to more than just ankle joint afferent losses.

The clinical application of these results directly relates to ankle injury prevention and rehabilitation programs. Although much focus in the last few decades has been centered on studying the etiology of functional ankle instability, definitive factor(s) remain elusive. One of the prominent thoughts has been the idea that "articular deafferentation" accompanying inversion ankle injury is responsible for producing the signs and symptoms associated with functional ankle instability (9). The experimental methodology of the current investigation provided a method by which articular deafferentation could be temporarily induced, in isolation, without disrupting the mechanical stability or motor abilities of the ankle joint. The lack of significant changes after anesthesia induction provides an objective rationale for clinicians to consider other components of the postural control system during ankle injury prevention and management. In addition, these results suggest there is potential for normal postural control to exist after injury in persons with an otherwise unremarkable ankle joint (i.e., strength, mechanical stability).

Similar experimental mechanoreceptor manipulations have been previously used $(3,7,12,18)$. In contrast to the two previous investigations considering the contribution of ankle ligament mechanoreceptor inputs for postural control $(3,12)$, it was decided to inject both the calcaneofibular and anterior talofibular ligament regions because of the frequency both ligaments become damaged during inversion ankle sprains.

Single leg postural control was the focus of this investigation because of the popularity for which it is used during orthopedic clinical evaluations and research. Additionally, periods of single leg stance are often interspersed within many functional activities. We speculated that constraining the postural control system to maintaining equilibrium over a single, short, and narrow base of support would likely rely more heavily on ankle somatosensory inputs. In the context of the current investigation, this was speculated to increase the effect size of the lateral ankle ligament anesthesia.
Unique to the present investigation was the incorporation of the traditionally used SLEO and SLEC tasks, as well as a more dynamic postural control task. Using these tasks allowed for studying the steady-state characteristics involved with postural control (8). Within the context of the present investigation, the single leg landing provided a method to more closely recreate the forces and postural demands incurred during functional activity while slightly shifting the conscious attention away from sole concentration on maintaining equilibrium.

Partly in anticipation of subtle treatment effects, a multivariate postural control measurement approach incorporating the combination of kinematic and forceplate variables was utilized. The other rationale for incorporating a kinematic model was the work published by Tropp and Odenrick (29). In their investigation, they revealed that persons with functional ankle instability displayed an increased tendency to use more postural movements at the hip-trunk, rather than the ankle in comparison to healthy participants. The tendency of persons with functional ankle instability to rely more heavily on the hip-trunk for corrective action has also been demonstrated in a subsequent report by the same authors (10), as well as another group of authors using postural perturbations (21). Based on these two studies, it had been hypothesized that increased reliance on the knee and hip joints would have become apparent after the anesthetic treatment during all three postural control tasks. The nonsignificant joint by condition interactions for the quantity of joint corrective action across each of the postural control tasks refute this hypothesis.

In addition to lateral ankle ligament mechanoreceptors, afferent information for postural control also arises from other mechanoreceptors within the somatosensory system, as well as from visual and vestibular sources. Specific to the ankle, this would include the talocrural and subtalar joint capsule and remaining ligamentous mechanoreceptors, muscle spindles embedded within each muscle crossing these joints, and the plantar cutaneous mechanoreceptors. Several studies have demonstrated that in circumstances where one or two sensory modalities are deficient, sufficient compensatory information can be provided by the remaining source(s) for equilibrium to be maintained $(4,13,19)$. With respect to the present investigation, vision was controlled by completing trials under eyes open and eyes closed conditions. The remaining sources, vestibular and other mechanoreceptors within the somatosensory system, were not experimentally manipulated but rather held constant across the treatment conditions. Vision was chosen for manipulation because of the potent influences demonstrated during

TABLE 6. Means $( \pm S D)$ for joint corrective action, stabilization time, and center of mass variables during SLLand.

\begin{tabular}{|c|c|c|c|c|c|}
\hline & \multicolumn{3}{|c|}{ Joint Corrective Action $\left({ }^{\circ}\right)$} & \multirow[b]{2}{*}{ STAB (s) } & \multirow[b]{2}{*}{$\operatorname{COM}_{\text {Height }}(\%)$} \\
\hline & Ankle* & Knee† & Hip-Trunk & & \\
\hline $\begin{array}{l}\text { Control } \\
\text { Anesthesia }\end{array}$ & $\begin{array}{l}0.2600 \pm 0.0523 \\
0.2618 \pm 0.0488\end{array}$ & $\begin{array}{l}0.3455 \pm 0.0668 \\
0.3484 \pm 0.0664\end{array}$ & $\begin{array}{l}0.1329 \pm 0.0409 \\
0.1248 \pm 0.0343\end{array}$ & $\begin{array}{l}1.115 \pm 0.081 \\
1.143 \pm 0.082\end{array}$ & $\begin{array}{l}98.5 \pm 2.1 \\
99.7 \pm 4.5\end{array}$ \\
\hline
\end{tabular}

STAB, stabilization time; $\mathrm{COM}_{\text {Height }}$, normalized vertical center of mass distance from ankle joint.

* Ankle significantly greater than hip-trunk ( $P<0.05$, main effect for joint).

$\dagger$ Knee significantly greater than ankle and hip-trunk $(P>0.05$, main effect for joint). 
single leg stance (26) and the ease with which it can be eliminated (eye closure). Vestibular inputs have been attributed with operating at low frequencies, functioning primarily as static vertical references and providing the inputs necessary for precise and independent control of head and eye positions (20). In light of these roles, vestibular inputs were left constant through the investigation due to the difficulty surrounding the manipulation of vestibular inputs. In contrast to the seemingly subtle role of vestibular inputs, the contribution of somatosensory sources not manipulated in this investigation, especially the muscle spindles, has been widely demonstrated $(5,6,8,22)$. Similar to vestibular inputs, experimentally eliminating individual somatosensory organs in isolation is extremely difficult and, therefore, was not addressed in the present investigation. Thus, a potential explanation of the nonsignificant results could simply be that the remaining sensory inputs (vestibular and remaining somatosensory receptors) were sufficient to completely compensate for the disruptions induced by the treatments $(4,13,20)$. If the compensatory theory is further entertained, independent of the contributing source(s), it would suggest that individually the lateral ankle articular mechanoreceptors are either not important for postural control or that they do not have a unique, irreplaceable role within the context of the postural control tasks utilized in this investigation.

Another plausible explanation for the nonsignificant results may be that despite the efforts to utilize tasks and measurement methods believed to be sensitive to even subtle differences, the contribution of the targeted mechanoreceptors still remained elusive. In other words, the contribution of ankle ligament mechanoreceptors may be more subtle and/or indirect. Johannson and colleagues (15) have suggested that articular mechanoreceptors exert potent influences upon gamma motor neurons responsible for muscle spindle sensitivity adjustment, which in turn regulates the muscle stiffness available for joint stability. Thus, the effect of the lateral ligament mechanoreceptor losses may possibly become more evident during tasks and/or using measurement techniques other than those utilized in this investigation. For example, all subjects in this investigation were given a practice session of each task before testing, and, therefore, were familiar with the expectations and the temporal/spatial alpha and gamma motor neuron activation requirements. In contrast, if subjects were not given a previous exposure to the tasks, the role of the targeted mechanoreceptors in motor learning/skill acquisition may have become more evident as the ligament mechanoreceptors would not have been available to provide their "perspective" to the task demands.

With respect to the forceplate variable of postural stability during SLEO and SLEC after lateral ankle anesthesia, the results of the current investigation parallel those of Hertel et al. (12). Both studies failed to reveal significant differences in the variation of the center of pressure around a mean location.

The kinematic model utilized in this investigation incorporated three segments, lower leg, upper leg, and trunk, using an electromagnetic tracking device. This was similar to the link-segment model utilized by Tropp and colleagues $(10,29)$, with the exception that we also monitored knee flexion. A limitation to the approach used in this investigation was the indirect measurement of ankle joint movements through tibial motion. Similar assumptions have been made $(10,29)$. This method, similar to our previous work $(26)$, was employed in the present investigation because of the distortion imposed on the electromagnetic field by the forceplate. Based on our own work examining this issue, the assumption that tibial motion reflects ankle motion during single leg stance appears to be valid provided the foot remains fixed and in contact with the support surface. By using this method, however, the possibility exists that some ankle motion went undetected thereby influencing our results.

Another potential explanation for the nonsignificant kinematic results after both treatments could have been that the subjects adopted altered mean joint positions during the postural control tasks. In other words, subjects could have assumed a posture of increased knee flexion and/or altered ankle and hip angles during the postural tasks after the anesthesia treatment. These altered mean angular positions could have enabled them to remain relatively stable, as demonstrated by no difference in the quantity of joint corrective action, despite diminished articular afferent information. However, disputing this idea was the lack of significant alteration in the $\mathrm{COM}_{\text {height }}$ between the anesthesia and control conditions. Interestingly, the participants did not alter their $\mathrm{COM}_{\text {height }}$ during the SLEC trials despite the increase in instability revealed by the quantity of corrective action, $\mathrm{COP}_{\text {Dist }}$, and compensatory event variables. This would suggest that lowering the $\mathrm{COM}_{\text {height }}$ may not be a strategy used when visual information is not available during single leg stance.

Although single leg postural control testing was desirable from the perspective of considering the significance of ankle articular inputs, the reduction in a person's base of support makes compensatory actions and falls frequent occurrences even in the absence of experimental manipulation or pathology (26). A number of methods have been used to manage this issue; however, they all appear to reduce the ability to make an accurate assessment and/or detect slight alterations in postural stability. Similar to our previous work (26), the approach chosen in this investigation was to include trials with compensatory events by truncating the measurement data during the event based on the definitions of the Balance Error Scoring System (BESS) (24). Using the BESS, the number of compensatory events occurring within each complete trial (defined as less than three compensatory events), time of first compensatory event, and total time of compensatory events for the SLEO and SLEC tasks became additional dependent variables. Results of the statistical analyses conducted on these variables failed to reveal significant differences attributable to a loss of lateral articular afferent information. This would support the notion that truncating the measurement data during compensatory events of each complete trial did not bias the kinematic and forceplate measures of postural stability. Similar to $\mathrm{COM}_{\text {height }}$, vision was revealed to have a potent impact on the compensatory 
event variables. Significant differences were revealed for both the number of compensatory events and the time to first compensatory event, whereas the total time of compensatory events approached significance.

Specific to the SLLand, it was hypothesized that the lateral ankle ligament anesthesia might alter both the preparatory and balance phases of the landing. Preparatory actions were considered by measuring angular position of the knee and hip-trunk ankle at ground contact. The angular position of the ankle would also have been an important addition; however, the limitations of the electromagnetic device with the forceplate prevented this consideration. There were no significant differences in joint angles at ground contact between the two conditions. After ground contact, $v_{G R F} F_{\text {Peak }}$ and $\mathrm{vGRF}_{\text {Time Peak }}$ were calculated. Similar to joint angle at ground contact, results of the statistical analyses for these variables failed to reveal significant differences, further supporting the idea that similar landing strategies were used during the two conditions. After ground contact during the SLLand, STAB Time $_{\text {and the quantity of }}$ joint corrective action during $\mathrm{STAB}_{\text {Time }}$ were calculated. Again, no significant treatment conditions differences were revealed. Thus, similar to the SLEO and SLEC, loss of lateral ankle articular mechanoreceptors did not have a significant effect on postural stability.

The potent effects of vision on joint corrective action demonstrated in this investigation are not that profound given the number of previous investigations that have established the influence of visual conditions on postural sway $(4,8)$. The significant vision by joint interactions and main effects for vision and joint during the static tasks are similar to the findings of our previous work comparing single leg postural control on firm, foam, and multiaxial support surfaces (26). Based on the results of both investigations, it appears that the ankle and hip-trunk joints are preferred over knee flexion as sources of corrective action during SLEC, whereas equal amounts of corrective action occur at the

\section{REFERENCES}

1. Bernier, J. N., D. H. Perrin, and A. Rijke. Effect of unilateral functional instability of the ankle on postural sway and inversion and eversion strength. J. Athl. Train. 32:226-232, 1997.

2. Colby, S. M., R. A. Hintermeister, M. R. Torry, and J. R. Steadman. Lower limb stability with ACL impairment. J. Orthop. Sports Phys. Ther. 29:444-454, 1999.

3. DeCarlo, M. S., and R. W. Tадвot. Evaluation of ankle proprioception following injection of the anterior talofibular ligament. J. Orthop. Sports Phys. Ther. 8:70-76, 1986.

4. Diener, H. C., and J. Dichgans. On the role of vestibular, visual and somatosensory information for dynamic postural control in humans. Prog. Brain Res. 76:253-262, 1988.

5. Diener, H. C., J. Dichgans, B. Guschlbauer, and H. Mau. The significance of proprioception on postural stabilization as assessed by ischemia. Brain Res. 296:103-109, 1984.

6. Dietz, V., G. A. Horstmann, and W. Berger. Significance of proprioceptive mechanisms in the regulation of stance. Prog. Brain Res. 80:419-423, 1989.

7. Feuerbach, J. W., M. D. Grabiner, T. J. Кoh, and G. G. WEIKER. Effect of an ankle orthosis and ankle ligament anesthesia on ankle joint proprioception. Am. J. Sport Med. 22:223229, 1994. three joints during SLEO. In contrast to the ankle and hip-trunk joints dominating corrective action during SLEO and SLEC, the most angular motion occurred at the knee after SLLand. This result is not that surprising considering the need for shock absorption after ground contact. Collectively, the kinematic results during the stance and landing tasks support the need for incorporation of multilink models during investigations of single leg stance.

One difference with the current investigation was that the trunk and hip were considered together, rather than separate joints. When considered together, this investigation revealed that similar quantities of corrective action occur at the ankle and hip-trunk for both SLEO and SLEC. Tropp and Odenrick (29) reported that that the quantity of hip-trunk corrective action in the frontal plane was greater than lower leg rotation, which was assumed to indirectly reflect ankle inversion-eversion, during SLEO. The difference between the current investigation and that of Tropp et al. relates to a three-dimensional model versus a two-dimensional model. The decision to use a three-dimensional vector approach was based upon pilot work revealing each joint motion separately (i.e., flexion-extension, abduction-adduction, and medial-lateral rotation) to be unstable across multiple trials, with the absolute and relative reliability for the three-dimensional vector approach being superior (26).

In conclusion, this investigation failed to demonstrate that lateral ankle ligament mechanoreceptors make notable contributions to single leg postural control during static and dynamic tasks. Within the context of the postural control tasks utilized in this study, the nonsignificant results suggest that either the targeted mechanoreceptors are not important for postural control or do not have unique, irreplaceable roles. These results contradict the traditional orthopedic perspective that after injury postural control becomes disrupted solely because of articular mechanoreceptors disruptions.

8. Fitzpatrick, R., D. K. Rogers, and D. I. McCloskey. Stable human standing with only lower-limb muscle afferents providing the only sensory input. J. Physiol. 480:395-403, 1994.

9. Freeman, M. Instability of the foot after injuries to the lateral ligament of the ankle. J. Bone Joint Surg. 47B:669-677, 1965.

10. Gauffin, H., H. Tropp, and P. Odenrick. Effect of ankle disk training on postural control in patients with functional instability of the ankle joint. Int. J. Sports Med. 9:141-144, 1988.

11. Guskiewicz, K. M., and D. H. Perrin. Effect of orthotics on postural sway following inversion ankle sprain. J. Orthop. Sports Phys. Ther. 23:326-331, 1996.

12. Hertel, J., K. M. Guskiewicz, D. M. Kahler, and D. H. Perrin. Effect of lateral ankle joint anesthesia on center of balance, postural sway and joint position sense. J. Sport Rehabil. 5:111-119, 1996.

13. Horak, F. B., L. M. Nashner, and H. C. Diener. Postural strategies associated with somatosensory and vestibular loss. Exp. Brain Res. 82:167-177, 1990.

14. Isakov, E., and J. Mizrahi. Is balance impaired by recurrent sprained ankle. Br. J. Sports Med. 31:65-67, 1997.

15. Johansson, H., P. Sjolander, P. Sojka, and I. Wadell. Reflex actions on the $\gamma$-muscle spindle systems of muscles acting at the 
knee joint elicited by stretch of the posterior cruciate ligament. Neuro-Orthoped. 8:9-21, 1989.

16. Konradsen, L., S. Olesen, and H. M. Hansen. Ankle sensorimotor control and eversion strength after acute inversion injuries. Am. J. Sports Med. 26:72-77, 1998.

17. Lephart, S. M., M. S. Kocher, F. H. Fu, P. A. Borsa, and C. D. HARNER. Proprioception following anterior cruciate ligament reconstruction. J. Sport Rehabil. 1:188-196, 1992.

18. Myers, J. B., B. L. Riemann, J. H. Hwang, S. M. Lephart, and F. H. Fu. Effects of peripheral afferent alteration of the lateral ankle ligaments on dynamic stability. Am. J. Sports Med. 31:498506, 2003.

19. NAshner, L. A model describing vestibular detection of body sway motion. Acta. Otolaryngol. 72:429-436, 1971.

20. Nashner, L., F. Black, and C. Wall. Adaptation to altered support and visual conditions during stance: Patients with vestibular deficits. J. Neurosci. 2:536-544, 1982.

21. Pintsaar, A., J. Brynhildsen, and H. Tropp. Postural corrections after standardized perturbations of single limb stance: effect of training and orthotic devices in patients with ankle instability. Br. J. Sports Med. 30:151-155, 1996.

22. Pyyko, I., H. Aalto, H. Seidel, and J. Starck. Hierarchy of different muscles in postural control. Acta. Otolarynogol. 468: 175-180, 1989.

23. RIEMANN, B. L. Is there a link between chronic ankle instability and postural instability. J. Athl. Train. 37:386-393, 2002.
24. Riemann, B. L., K. M. Guskiewicz, and E. W. Shields. Relationship between clinical and forceplate measures of postural stability. J. Sport Rehabil. 8:71-82, 1999.

25. Riemann, B. L., B. A. Munkasy, and M. J. Gale. Relationship between center of pressure and center of mass excursion during single leg stance equilibrium. J. Athl. Train. 37:S95, 2002.

26. Riemann, B. L., J. B. Myers, and S. M. Lephart. Comparison of the ankle, knee, hip and trunk corrective action shown during single-leg stance on firm, foam and multiaxial surfaces. Arch. Phys. Med. Rehabil. 84:90-95, 2003.

27. Stubblefield, E. S., B. L. Riemann, R. J. Schmitz, and J. B. Myers. Comparisons of ankle kinematic assessment approaches during single leg stance. J. Athl. Train. 36:S36, 2001.

28. Tropp, H., J. Ekstrand, and J. Gillquist. Stabilometry in functional instability of the ankle and its value in predicting injury. Med. Sci. Sports Exerc. 16:64-66, 1984.

29. Tropp, H., and P. Odenrick. Postural control in single-limb stance. J. Orthoped. Res. 6:833-839, 1988.

30. Wilkerson, G. B., J. J. Pinerola, and R. W. Caturano. Invertor vs evertor peak torque and power deficiencies associated with lateral ankle ligament injury. J. Orthop. Sports Phys. Ther. 26:78-85, 1997.

31. Winter, D. A. Biomechanics and Motor Control of Human Movement, 2nd Ed. New York: John Wiley \& Sons, Inc, 1990, pp. $56-57$. 